\title{
Imaging birefringent tissue in the human tympanic membrane by polarization-sensitive optical coherence tomography
}

\author{
Svea Steuer ${ }^{1, *}$, Jonas Golde ${ }^{1}$, Steffen Ossmann ${ }^{2}$, Lars Kirsten ${ }^{1}$, Joseph Morgenstern ${ }^{2}$, Matthias Bornitz ${ }^{2}$, Marcus \\ Neudert ${ }^{2}$, and Edmund Koch ${ }^{1}$ \\ ${ }^{1}$ Clinical Sensoring and Monitoring, Anesthesiology and Intensive Care Medicine, Technische Universität Dresden, Fetscherstrasse 74, \\ 01307 Dresden, Germany \\ ${ }^{2}$ Otorhinolaryngology, Carl Gustav Carus Faculty of Medicine, Technische Universität Dresden, Fetscherstrasse 74, 01307 Dresden, \\ Germany
}

\begin{abstract}
Acousto-mechanical properties of the human tympanic membrane mainly depend on the connective tissue in its layered structure. Using polarization-sensitive optical coherence tomography, a depth-resolved imaging technique which provides additional tissue specific contrast, polarization changes of the birefringent layers in the human tympanic membrane were detected. By depicting estimated local retardances, distinguishing different tissue types was possible. This suggests the ability to image pathological alterations of the connective tissue with PSOCT, which extends the conventional diagnostic methods in middle ear surgery.
\end{abstract}

\section{Introduction}

Normal hearing requires the acousto-mechanical conduction of sound waves and impedance matching in the middle ear, consisting of the tympanic membrane (TM) and the ossicles. Stability and movement properties of the TM are mainly defined by a connective tissue layer [1], which is embedded in between an epidermal layer of the ear canal and mucosal tissue of the middle ear. If pathological changes of the TM are present, e.g. in succession of a chronic otitis media, the oscillation behavior of the eardrum and thus the sound conduction of the middle ear is impaired which has a direct effect on hearing. In clinical practice, otoscopy provides a general overview of the semi-transparent eardrum and adjacent structures, which is supported by tympanometry and audiometry for the assessment of middle ear function. However, alterations of the layered TM structure are not accessible with these methods. Optical coherence tomography (OCT) is a non-invasive and three-dimensional imaging technique which has been demonstrated as a promising tool for middle ear diagnostics [2]. Conventional OCT measures the backscattered signal of a sample, but is limited in providing tissue-specific contrast. Polarization-sensitive OCT (PSOCT) additionally detects polarization-changing properties of a sample, i.e. birefringence and depolarization [3]. Here, it is shown that the connective tissue layer of the TM can be assessed by PSOCT due to its birefringent collagen fiber structure. Utilizing an algorithm for the visualization of local retardance, the layered TM structure of a temporal bone specimen is examined.

\section{Method and measurements}

To perform ex vivo PSOCT imaging of the TM, a right human cadaveric temporal bone, which was stored frozen after extraction, was prepared for measurements with a bench-top scanner by removing the auditory canal. A commercial spectrometer-based PSOCT system (Thorlabs TEL220PS) with a broad-bandwidth light source centered at $1300 \mathrm{~nm}$ and a telecentric scan lens (Thorlabs OCTLK3) was utilized. While the measurement and the acquisition of the raw spectra was done using the provided commercial software (Thorlabs ThorImage OCT), further data handling was implemented in Matlab, including OCT processing and polarization analysis. After spectral shaping, k-linearization and Fourier transforming of the raw spectra to obtain complex depth profiles for both polarization channels, Stokes vectors were calculated and spatially averaged using a 3D Gaussian kernel with a full width at half maximum of $30 \mu \mathrm{m}$ in axial direction and $60 \mu \mathrm{m}$ in both lateral directions. As the utilized PSOCT system measures the backreflected signal for a single circularly polarized illumination state with polarization-diverse detection, the acquired cumulative polarization contrast is highly depending on the arrangement and orientation of the subsequent layers in a complex sample. To derive the local sample retardance, we calculated the angle between the averaged Stokes vectors of subsequent pixels over depth. As the result is only a valid retardance for certain constellations including circularly illuminated layers, the presented measure is only an estimate of the local retardance. For qualitative evaluation of birefringent and non-birefringent TM layers, both intensity images and visualizations of estimated local retardance with overlayed intensity are presented (see Fig. 1).

*e-mail: svea.steuer@mailbox.tu-dresden.de 


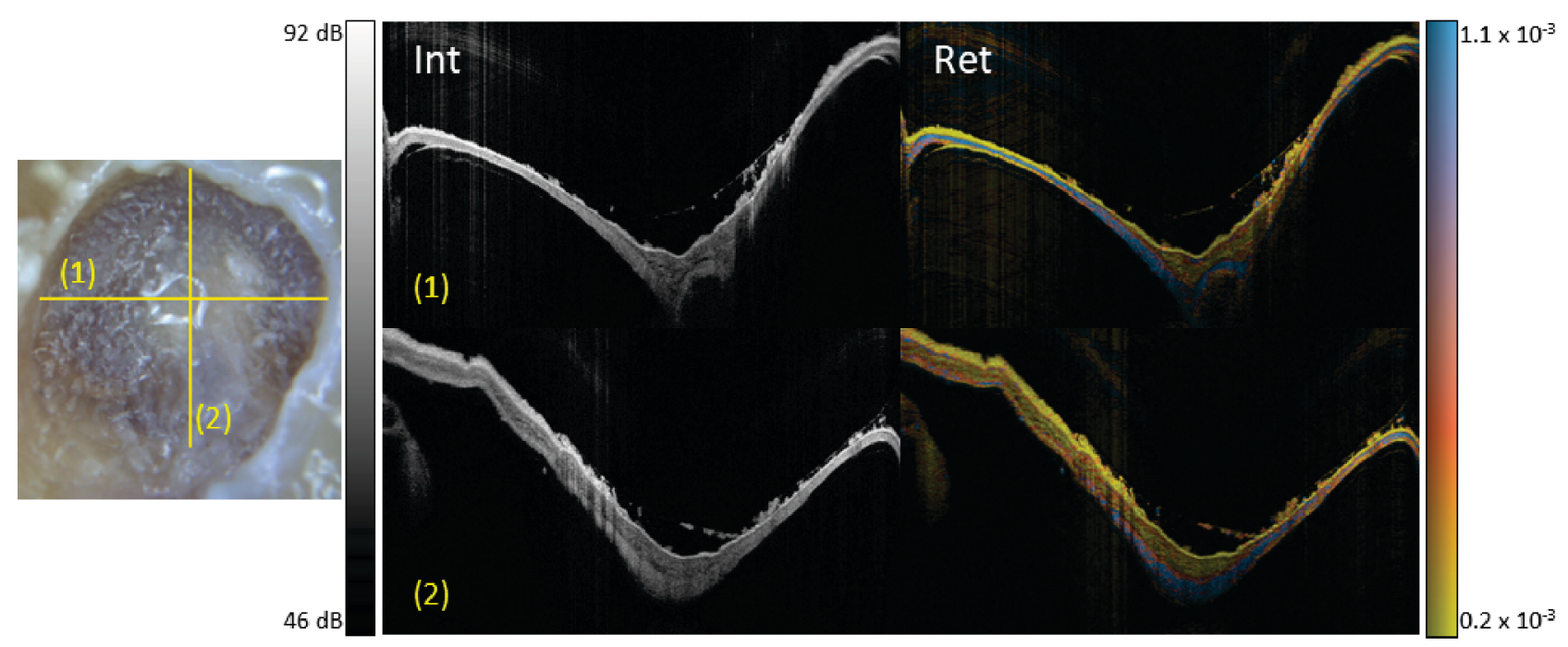

Figure 1. Photographic overview of the tympanic membrane (left) of a right temporal bone specimen with marked positions, (1) and (2), of the exemplary PSOCT B-scans. Intensity (middle column) and estimated local retardance (right column) images are $7.6 \mathrm{~mm}$ in lateral direction and $3.15 \mathrm{~mm}$ in axial direction.

\section{Results}

The connective tissue of the TM is commonly described as a two-layer arrangement of radially and circularly aligned collagen fibers surrounding the malleus. Although limited in the field of view, non-linear microscopic recordings of TMs, based on second harmonic generation, indicate that the fibrous structure of the connective tissue is interwoven in a more complex way. By using PSOCT and the estimated local retardance within the sample, the layered structure of the TM was assessed. The birefringent connective tissue causes local polarization changes which were detected and visualized by the colored contrast depicted in Fig. 1. Differentiation of the connective tissue with high retardance (blue) from the less or nonbirefringent epidermal and mucosal tissue (yellow) was possible thereby. This was particularly true for the outer regions of the membrane in both scanning directions. In contrast to the intensity images, not only structures but also different tissue types could be distinguished. Thus, the results indicate the direct coupling of the connective tissue to the handle of malleus. However, resolving finer structures, e.g. individual collagen fibers, was impossible due to the limited axial and lateral resolution as well as applied spatial Stokes averaging.

\section{Conclusion}

Visualizing the connective tissue of the human tympanic membrane was achieved by PSOCT and an algorithm for the qualitative evaluation of the local retardance. As different diseases affecting the human tympanic membrane and its acousto-mechanical properties are related to alterations of the fibrous structure, e.g. tympanosclerosis, distinguishing the collagen fiber layer from the embedding tissue might provide additional diagnostic value in comparison to conventional OCT.

\section{Acknowledgments}

The author Svea Steuer is supported by the European Union/European Social Fund (ESF) and the Free State of Saxony with a doctoral scholarship (Project No. 100380876). Temporal bone experiments were approved by the Institutional Review Board (IRB00001473) at TU Dresden (EK108032015).

\section{References}

[1] K.N. O'Connor, M. Tam, N.H. Blevins, S. Puria, The Laryngoscope 118, 483 (2008)

[2] L. Kirsten, M. Schindler, J. Morgenstern, M.T. Erkkilä, J. Golde, J. Walther, P. Rottmann, M. Kemper, M. Bornitz, M. Neudert et al., Journal of Biomedical Optics 24, 1 (2018)

[3] J.F. de Boer, C.K. Hitzenberger, Y. Yasuno, Biomed. Opt. Express 8, 1838 (2017) 